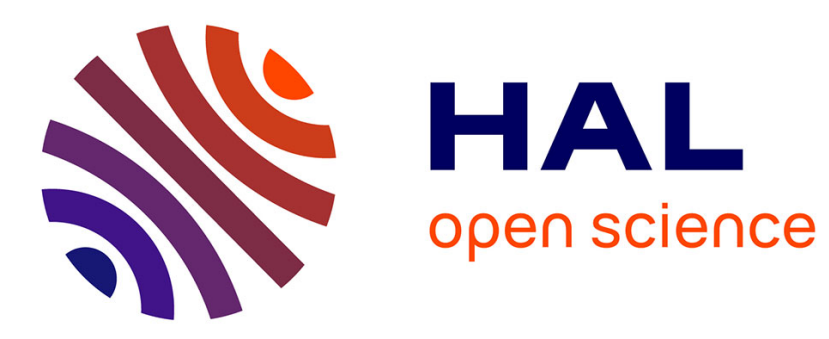

\title{
De la prévention au dépistage ou l'affirmation des médecins scolaires (1879-1939)
}

\author{
Mathias Gardet
}

\section{To cite this version:}

Mathias Gardet. De la prévention au dépistage ou l'affirmation des médecins scolaires (18791939). Informations sociales, 2010, Eduquer et prévenir: ce que fait l'école, 161, p. 14-21. 10.3917/inso.161.0014 . hal-01086480

\section{HAL Id: hal-01086480}

\section{https://hal-univ-paris8.archives-ouvertes.fr/hal-01086480}

Submitted on 13 Oct 2020

HAL is a multi-disciplinary open access archive for the deposit and dissemination of scientific research documents, whether they are published or not. The documents may come from teaching and research institutions in France or abroad, or from public or private research centers.
L'archive ouverte pluridisciplinaire HAL, est destinée au dépôt et à la diffusion de documents scientifiques de niveau recherche, publiés ou non, émanant des établissements d'enseignement et de recherche français ou étrangers, des laboratoires publics ou privés. 


\title{
De la prévention au dépistage ou l'affirmation des médecins scolaires (1879-1939)
}

\author{
par Mathias Gardet, historien, PR en sciences de l'éducation, université de Paris 8 \\ publié dans Informations sociales, $\mathrm{n}^{\circ} 161$, septembre-octobre 2010, p. 14-21
}

Le rapport entre médecins et institution scolaire se modifie profondément à la fin du XIX siècle avec la généralisation de l'inspection médicale au sein des établissements primaires. Des problèmes de santé, on passe progressivement à l'idée d'organiser la prévention et l'hygiène scolaire. Le médecin n'est plus là pour soigner les élèves qui seraient tombés malades mais comme garants de la santé physique et mentale de l'écolier sain et, au-delà, comme surveillant et conseiller de la salubrité des locaux scolaires. Ces visites, inscrites dans le cadre de tournées médicales, restent ponctuelles et, pendant longtemps, le médecin ne bénéficie pas de relais permanent au sein des établissements, ses seuls informateurs étant les instituteurs. Avec la montée en puissance des travaux impulsés par les premiers psychologues, notamment au sein du laboratoire de psychologie de la Sorbonne, ainsi que ceux donnant corps à la psychiatrie et à la neuropsychiatrie infantile, le rôle des médecins trouve une nouvelle dimension. L'école accueillant sans sélection les enfants issus des couches populaires devient en soi un champ d'expérimentation où l'on peut tester les nouvelles théories et constructions nosographiques et s'essayer à dépister pour les uns les enfants arriérés ou anormaux, pour les autres les futurs délinquants, quand on ne cherche pas à démontrer qu'il s'agit en fait d'une seule et même catégorie. Les qualifications requises pour ce dépistage reposent sur une science et une formation qui de plus en plus sera l'apanage du monde médical.

\section{À l'heure de l'hygiène scolaire}

Bien que largement débattue et préconisée durant tout le $\mathrm{XIX}^{\mathrm{e}}$ siècle, la présence des médecins dans les écoles primaires peine à s'organiser. La première édition de 1882-1893 du dictionnaire de pédagogie et d'instruction primaire ${ }^{1}$, consacre un article spécifique à "Inspection médicale des écoles » qui montre encore à quel point elle est balbutiante malgré la circulaire du ministre de l'Instruction publique aux préfets, en date du 14 novembre 1879, qui prescrivait de généraliser dans toute la France un service de médecins inspecteurs à l'image de ceux organisés à Paris et dans quelques grandes villes. Il est ainsi rappelé qu'il s'agit du premier et unique document officiel relatif à cette importante question. Dans cette circulaire, le ministre Jules Ferry souligne que cette fonction de surveillance des bonnes conditions sanitaires des locaux scolaires était assurée par les inspecteurs primaires qui malgré "leur zèle et leur vigilance, ne possèdent en général que des connaissances médicales imparfaites, et certaines circonstances susceptibles d'influer sur la santé de la population infantile peuvent leur échapper». S'il se plait à penser que son "projet ne rencontrera dans l'application aucune difficulté sérieuse », il se retrouve bien obligé encore à employer le conditionnel pour formuler son souhait: "Ily aurait dans chaque canton un ou plusieurs médecins chargés de visiter, dans leurs tournées de clientèle, les écoles publiques, au double point de la salubrité des bâtiments et de l'état sanitaire des élèves. »Une des raisons de ce conditionnel peut s'expliquer par le flou qui subsiste quant au financement d'une telle opération, Jules Ferry se contentant de préconiser "une légère rétribution » qui " pourrait au besoin être votée par les communes intéressées». De fait, l'article du dictionnaire, rédigé dix ans après cette circulaire, montre que l'optimisme affiché par Jules Ferry s'est heurté à

\footnotetext{
1. Dubois P., 2000, «Le Dictionnaire de F. Buisson et ses auteurs (1878-1887) », Histoire de l'éducation, n 85 , p. 2-17.
} 
des difficultés d'application, puisque seuls ont répondu à l'appel le département de la Seine qui a créé 114 places de médecins inspecteurs et la ville de Lyon qui a fondé un service d'inspection médicale dès le $1^{\text {er }}$ janvier 1880.

\section{Hygiène de l'ecole et hygiène de l'écolier}

Le rôle de ces médecins ne se cantonne pas dans la détection des maladies, on peut même être surpris, en lisant l'article de ce même dictionnaire consacré aux «maladies », de découvrir que ce devoir incombe essentiellement à l'instituteur qui, sans évidemment poser de diagnostic, doit savoir reconnaittre certains symptômes et pouvoir ainsi « refuser l'entrée de l'école à tout enfant qu'il soupçonne atteint d'une maladie contagieuse »; au médecin de famille de prendre ensuite le relais. Le médecin a en revanche une place centrale dans la question de l'hygiène scolaire, dont il est depuis le début du $\mathrm{XIX}^{\mathrm{e}}$ siècle un des principales théoriciens et qui recouvre aussi bien l'hygiène des locaux (bâtiments, salles de classe, salles d'études, réfectoire, cour, infirmerie, latrines), l'hygiène physique de la personne (soins corporels, vêtements, boissons, aliments, horaires des repas, temps consacré au sommeil, aux récréations, type d'études) et l'hygiène morale (ordre moral et religieux, discipline $)^{2}$. Dans le très long article consacré à cette question dans la première édition du dictionnaire de pédagogie est ainsi développé sur le même plan l'hygiène de l'école avec un rappel très détaillé des règles édictées entre autres par un arrêté ministériel du 17 juin 1880 (sur l'emplacement des locaux, le terrain, l'exposition des bâtiments, les cours et préaux, le gymnase, le vestiaire, le mobilier scolaire, les cabinets d'aisance...) et l'hygiène de l'écolier qui précise les conditions d'admission, relègue le thème des maladies scolaires à l'article spécifique qui leur est consacré, pour s'intéresser en détail aux notions de propreté, d'alimentation, de temps de récréations, de gymnastique et même s'attarder sur les punitions. Si l'instituteur reste le principal exécutant de cette mise aux normes, seul le médecin est considéré « apte à juger » :

"Ceci nous amène à l'une des questions les plus importantes, parmi celles qui touchent à l'bygiène de l'école, celle de l'inspection médicale. La surveillance exercée par l'homme de l'art s'impose de plus en plus, à l'État et aux communes, comme l'une des premières mesures préservatrices à organiser. Nous savons qu'elle existe dans quelques grandes villes, à Paris en particulier: mais là même elle est incomplète et n'offre pas toutes les garanties désirables. Il faudrait qu'elle constituât partout un service régulier, consistant en visites périodiques, contrôlées par des registres et par des rapports à l'appui. Ce serait là le seul moyen de soulager l'instituteur d'une responsabilité qui dépasse ses forces et de lui permettre de s'abriter derrière l'autorité médicale pour réaliser plus sûrement les réformes nécessaires. »

\section{Extension du rôle du médecin-inspecteur}

Dans la seconde édition du dictionnaire de pédagogie et d'instruction primaire parue en 1911, le docteur L. Dufestel, secrétaire général de la Société d'hygiène scolaire et médecin inspecteur des écoles de Paris, est chargé de rédiger un long article sur le «Médecin inspecteur » dans lequel il se félicite de la consécration et de «l'extension considérable» qu'a connu entre temps cette fonction. Il n'évoque en fait dans son texte que l'exemple de la ville de Paris où le service a été réorganisé en profondeur et renforcé, le nouveau règlement de la Capitale précisant que « chaque médecin a environ 1000 élèves à surveiller », qu'il « doit faire une visite par semaine à jours et heures fixés d'accord avec l'administration». Après la visite des classes, il se rend "dans la pièce qui lui est réservée» pour examiner «tous les enfants suspects ou malpropres» qu'il aura ou que l'instituteur lui aura désigné, ainsi que tous ceux «qui ont été absents de l'école sans motifs connus pendant plus de trois jours ». Il doit enfin, pour chaque élève nouveau constituer une fiche sanitaire. Si l'on retrouve dans ces indications la politique prophylactique de lutte contre les

\footnotetext{
2. Tschirhart A., 2008/2, «Rôle et évolution de l'hygiène scolaire dans l'enseignement secondaire de1800 à 1910 ", Carrefours de l'éducation, $\mathrm{n}^{\circ} 26$, p. 201-213
} 
épidémies, notamment contre la tuberculose, le Dr Dufestel insiste bien sur le fait qu'il ne s'agit pas du seul rôle octroyé au médecin inspecteur :

«Créé primitivement pour protéger la collectivité des écoliers contre les maladies, le médecin scolaire devint peu à peu, et par la force des choses, le surveillant autorisé du développement physiologique de l'écolier pris individuellement.»

Il évoque à nouveau l'importance de l'examen hygiénique des bâtiments et du mobilier scolaire, mais aussi la vigilance critique que doit déployer le médecin inspecteur envers «les programmes trop chargés, le surmenage, et surtout le malmenage, si nuisible au développement de l'enfant».

Il est cependant précisé que cette intervention des médecins dans l'école ne peut se faire sans une étroite collaboration avec les instituteurs. Comme le rappelle un autre article du dictionnaire consacré aux "Infirmières d'école primaire», les médecins français, contrairement à leurs homologues anglais ou américains ne disposent pas encore de ces «auxiliaires précieuses » que sont les infirmières d'école ou "school nurses». Malgré un essai tenté dans les deux écoles de filles du deuxième arrondissement de Paris en 1908 avec deux infirmières de l'École de la Salpêtrière, ce type d'expériences se heurte semble-t-il en France à la résistance du milieu scolaire et des parents d'élèves. Ce n'est que très timidement qu'elles apparaitront au compte-goutte dans certaines écoles durant l'entre-deux-guerres avec l'affirmation du métier d'infirmière ${ }^{3}$, l'histoire de leur implantation en milieu scolaire est d'ailleurs encore très largement méconnue.

\section{Dépistage et rendement social}

Parallèlement à cette montée en puissance des médecins-inspecteurs sur les questions d'hygiène scolaire, commencent à se diffuser les travaux menés au sein du laboratoire de psychologie physiologique de la Sorbonne créé en 1889 par le médecin Henry Beaunis et rejoint en 1891 par Alfred Binet qui en prendra la direction à partir de $1894^{4}$. Ce dernier, bien que fils de médecin, n'avait pas repris pas le flambeau, il avait entamé des études juridiques, puis des études de sciences naturelles qui l'avaient amené à soutenir une thèse de doctorat ès sciences sur le système nerveux sous-intestinal des insectes. Passionné de psychophysiologie, il développe avec ses collaborateurs l'étude des processus psychiques et de leurs variations d'un individu à l'autre par le biais d'épreuves simples, variées, rapides qui ne nécessite pas d'appareillages sophistiqués. Faute de trouver suffisamment de sujets au sein du laboratoire qui reste peu fréquenté et n'ayant pas réussi à pénétrer les collèges et lycées, il se rabat sur l'école primaire où il obtient toutes les autorisations.

\section{Des classes adaptées aux « arriérés »}

En 1899, ses expériences connaissent un nouvel essor par le tandem désormais inséparable qu'il constitue avec le jeune médecin-psychiatre Théodore Simon, directeur de la colonie d'enfants arriérés du Perray Vaucluse. Ils travaillent en commun à l'asile et dans une école du quartier populaire de Belleville où ils élaborent, en 1905, une première version de leur échelle métrique de l'intelligence qui propose une mesure basée sur une classification subtile entre âge réel et âge mental, détectée selon un test devenu célèbre portant le nom de ses deux inventeurs (ce test sera repris et transformé plus tard sous la forme du test du quotient intellectuel ou Q.I. $)^{5}$. Malgré la

\footnotetext{
3. Collière M-F., Diebolt E. (dir.), 1988, Pour une histoire des soins et des professions soignantes, Sainte-Foy-lès-Lyon, Amiec, cahier n`10, 299 p.; Kniebielher Y., Leroux-Hugon V. et al., 1984, Cornettes et blouses blanches : les infirmières dans la société française, 1880-1980, Paris, Hachette littérature, 366 p.

4. Nicolas S., 1995, «Henry Beaunis (1830-1921) directeur-fondateur du laboratoire de Psychologie physiologique de la Sorbonne », L'année psychologique, vol. 95, n². pp. 267-291.

5. A. Ohayon, «Entre gloire et opprobre, modeste éclairage historique sur la construction de l'échelle d'intelligence de Binet et Simon ", communication au colloque" Alfred Binet e la misura dell'intelligenza", 8-9 mai 2009, Urbino, Italie (à paraître).
} 
fonction occupée par Théodore Simon, ils prennent le contre-pied des orientations données par les médecins aliénistes comme Désiré Magloire Bourneville qui défendaient l'éducation à tout prix d'enfants jusqu'alors considérés comme incurables (idiots, épileptiques...), en fondant des asiles-écoles ou faute de mieux en proposant de les intégrer dans le système scolaire par la création de classes adaptées. A. Binet et T. Simon opposent à ces tentatives la notion de rendement social, s'insurgeant contre le coût et l'investissement de telles méthodes pour des sujets dont les progrès sont peu perceptibles et en tout cas insuffisants pour leur donner une réelle autonomie et leur permettre plus tard de «rembourser leur dette morale » en se rendant utiles à la société. Il préconisent alors d'abandonner l'apprentissage scolaire des enfants internés dans les asiles et de se contenter de leur inculquer les rudiments pour qu'ils puissent exercer les professions qui leur restent accessibles : la domesticité de province pour les "filles débiles ayant de bons instincts», les travaux agricoles pour les garçons, "car à la campagne la vie est moins compliquée, l'adaptation plus facile que dans les villes»" Ils proposent en revanche d'opérer un tri parmi les enfants scolarisés afin de repérer les anormaux d'école et, selon la même règle du rendement social, d'écarter ceux dont on ne peut espérer de progrès notables ou bien de créer des classes spéciales pour suivre ceux qui présentent un décalage avec les autres élèves sans préjuger de leurs capacité futures :

"Arrivons maintenant à nos anormaux d'école. On devine quelles vont être nos conclusions. Nous demandons expressément que leur rendement puisse être établi rigoureusement, et que les professeurs de ces écoles, et les inspecteurs, soient astreints à fixer exactement l'état mental et le degré d'instruction des élèves à leur entrée d'abord, à leur sortie ensuite. De cette manière, on opérera comme tout bon commerçant qui considère qu'un de ses premiers devoirs est de se rendre compte de ce qu'il fait; son système de comptabilité met dans ses affaires la clarté indispensable pour qu'il ne perde pas d'argent. Il sait à quel prix il achète, à quel prix et dans quelles conditions il vend, et si, par conséquent, ses profits sont suffisants pour l'encourager à continuer le commerce de tels et tels articles. ${ }^{7} »$

\section{De la sélection des élèves}

C'est leur vision des choses qui s'impose lors des premières expériences de classe de perfectionnement, comme celle tentée rue Belzunce à Paris dès 1907, qui seront consacrées deux ans plus tard par la loi du 15 avril 1909 «relative à la création de classes de perfectionnement annexées aux écoles élémentaires publiques et d'écoles autonomes de perfectionnement pour les enfants arriérés ». Binet et Simon entendent alors mettre en œuvre de façon systématique pour ce tri leur échelle d'intelligence, tout en préconisant la prudence dans son utilisation. S'ils évoquent l'importance pour effectuer ce dépistage de procéder à un examen médical et s'ils insistent sur le rôle que doit tenir le médecin dans la mise en place de ces classes ou écoles spéciales, par contre ils se montrent étonnamment réticents à lui octroyer toutes les attributions pour en piloter l'opération. Ils insistent ainsi sur le filtre premier que doivent continuer à exercer les instituteurs et les inspecteurs d'académie pour désigner les élèves à problème susceptibles de rejoindre ces classes et écoles de perfectionnement:

«Certaines questions échappent au médecin en tant que médecin pour être de la compétence soit du pédagogue, soit du psychologue. Mais il en est d'autres sur lesquelles aucune autre personne ne pourrait le remplacer. Limiter son rôle n'est pas l'amoindrir, bien au contraire; c'est lui assurer une autorité de bon aloi. Il n'a pas à choisir les anormaux parmi les normaux. Il a, chez les anormaux préalablement choisis, à différencier certains types et à prescrire certaines mesures soit d'admission, soit de conduite. ${ }^{8}$ "

Malgré les réserves exprimées, A. Binet et T. Simon ne tendent pas moins à favoriser l'affirmation du pouvoir des médecins scolaires déjà présents dans les établissements du primaire sur les questions d'hygiène et de Santé publique ainsi que le montre l'article déjà cité du docteur L.

\footnotetext{
${ }^{6}$. A. Binet, T. Simon, 1907, Les enfants anormaux, Paris, Librairie Armand Colin, p. 194.

7. Ibid., p. 192-193.

8. Ibid., p. 126.
} 
Dufestel, sur le «Médecin inspecteur» dans la seconde édition du dictionnaire de pédagogie et d'instruction primaire de 1911. Outre les compétences déjà évoquées, ce dernier attribue ainsi au médecin-inspecteur la fonction de détection des écoliers paresseux, des myopes et des demisourds et celle de "débarrasser la classe des anormaux psychiques qui, sans aucun profit pour eux, gênent le maître et retardent les progrès de leurs camarades. » Il revendique même pour ces médecins la paternité de la loi du 15 avril 1909.

\section{Anormaux-délinquants, une histoire de pourcentage}

La classification de type psychotechnique ou pédagogico-scientifique est finalement relayée par le diagnostic médico-psychiatrique; d'autant que dans le tandem Binet-Simon, c'est Alfred qui meurt le premier, d'une embolie cérébrale, en 1911. Un des signes de l'avènement de ce type d'évaluation et de l'arrivée de nouveaux experts peut se lire dans la thèse du médecin psychiatre Georges Heuyer soutenue en 1912 et publiée en 1914․ Il préconise comme Alfred Binet de procéder à une sélection parmi les élèves entre normaux et anormaux et parmi ces derniers entre éducables et inéducables, mais il pose avec détermination le rôle pivot et désormais incontournable que doit exercer le médecin dans le dépistage scolaire lié à la loi du 15 avril 1909. Pointant l'insuffisance de la fiche médicale individuelle classique rempli par le médecin inspecteur, il prêche pour l'établissement de carnets ou même de dossiers médico-pédagogiques individuels et insiste tout particulièrement sur la nécessité de procéder à un examen psychiatrique de l'écolier; examen qui lui semble d'autant plus indispensable que, comme l'indique le titre de sa thèse, il affirme le lien étroit existant entre la catégorie des anormaux et celle des jeunes délinquants selon une équation qu'il soutiendra tout le long de sa longue carrière : $80 \%$ des délinquants sont des anormaux et la plupart des anormaux, du fait de leur anormalité, sont des délinquants en puissance :

"Il est impossible de séparer théoriquement et pratiquement écoliers anormaux et délinquants juvéniles; ceux-ci se recrutent parmi ceux-là. C'est dès l'école qu'il faut essayer de faire le diagnostic des divers formes d'anomalie mentale : il faut pour chaque élève suspect, établir une fiche complète. ${ }^{10} "$

Faisant la synthèse des différentes méthodes expérimentées par la psychiatrie infantile naissante, il propose une fiche d'observation type, qu'il a l'occasion de tester lui-même auprès des écoliers, après sa nomination en 1920 comme médecin inspecteur des écoles de la Seine. Ses archives ${ }^{11}$ montrent l'influence grandissante de ses théories dans l'entre-deux-guerres, par la visibilité qui leur est données dans les principales revues médicales et de psychiatrie infantile, les colloques et autres événements organisés dans ce domaine et les très nombreuses conférences et formations proposées aux instituteurs et aux médecins scolaires de l'époque. Cette source foisonnante et d'une grande richesse reste encore très largement à explorer.

Entre la fin du XIX ${ }^{\mathrm{e}}$ siècle et les années 1930, on assiste ainsi à l'affirmation des médecins scolaires dont les attributions se trouvent démultipliées, non pas tant par leur action dans le domaine du soin et de la guérison, mais de par leur intervention généralisée auprès des écoliers sains dans une compréhension de plus en plus élargie de la notion de prévention ${ }^{12}$. D’une politique de prophylaxie contre les épidémies, on est passé à une conception très vaste de l'hygiène et de la santé de l'écolier qui va jusqu'à englober l'organisation du temps et de la

\footnotetext{
9. G. Heuyer, 1914, Enfants anormaux et délinquants juvéniles. Nécessité de l'examen psychiatrique des écoliers, Paris, G. Steinheil. 
discipline scolaires; d'un tri qui se voulait au départ très ciblé entre les enfants normaux et anormaux; les arriérés éducables et les inéducables, on est passé à un dépistage étendu aux indisciplinés et aux "pré-délinquants", faisant référence à des concepts pédagogiques et juridiques plutôt flous. Pour répondre à ces nouvelles sollicitations le médecin scolaire doit se doter de nouvelles compétences : de médecin généraliste, il doit être en plus fin psychologue, pour finalement se spécialiser en psychiatrie infantile. 